\title{
THE CASE FOR AND AGAINST PATIENT-HELD RECORDS
}

\author{
Chris McCann, MacMillan Nurse \\ St John's Hospice, Lancaster
}

\section{INTRODUCTION AND BACKGROUND}

The Calman Hine (1995) Report, A Policy Framework for Commissioning Cancer Services ${ }^{(1)}$, emphasises the importance of good communication between all those who care for cancer patients, and stresses the need for appropriate procedures to be set up to facilitate the communication process. Taking this into consideration, in 1995 I undertook a study to examine existing practices in communication between specialists working in cancer care and GPs. The aim of the study was to discover any problematic areas within the service, such as any failures or delays in the transmission of information, and any misunderstandings that may occur. What emerged from the data was an apparently simple idea which could minimise these problems and at the same time give the patient some ownership of and involvement in the decision-making processes and reduce the chance of ambiguities, misunderstandings and delays arising. That idea was to provide cancer patients with their own records, which they could take with them wherever they had contact with health professionals. The UKCC $(1993)^{(2)}$, is in favour of patients being given custody of their own health records in appropriate circumstances. They state that patient-held records (PHRs) help to emphasise and to make clear the practitioner's responsibility to the patient by sharing any information or assessments made and illustrate the involvement of patients in their own care.

A literature search at that time showed very little in the way of similar projects, except for the established use of PHRs issued to every child born in the UK and some studies on their use in the fields of obstetrics and mental health. There was also the accepted practice of issuing record cards for the use of patients with diabetes and those using anticoagulants. Little was published, however, describing the more comprehensive use of PHRs for cancer patients.

When a patient is diagnosed with cancer, his/her reactions to the diagnosis and the subsequent management can be extreme and have devastating effects on both the patient and the family ${ }^{(3)}$. Good communication between all health professionals involved in the patient's care is therefore vital to ensure that he/she receives a seamless and comprehensive service which involves as little misunderstanding and ambiguity as possible. A patient with cancer may be seeing a large number of different health professionals, others from allied professions and volunteers. Care provision must be carried out sensitively, maintaining confidentiality and with an acute awareness of the uniqueness of each individual. The Calman Hine Report (1995) states the importance of communication between primary care and specialised services, emphasising that communication should be appropriate in both time and content. Procedures should be set up in order to establish this wherever the patient may be during the course of the illness - home, hospital, GP surgery, hospice or any other setting where contact with a health professional is possible. Interdisciplinary teamwork is a vital part of cancer care, providing a comprehensive perspective in end-of-life or chronic illness ${ }^{(4)}$.

\section{PILOT STUDY AND EVALUATION}

A pilot study was carried out to introduce PHRs to three Lancaster GP practices, following consultations with the cancer steering group and the majority of the health professionals who would be involved. After the first 50 patients in the pilot study had used the records, I carried out an evaluation on their effectiveness. This evaluation consisted of some interviews with health professionals and a questionnaire to all the health professionals who had made an entry in the records, which had been returned to me after the period of care or when the patient had died. The returned records were examined closely to discover how they had been used and by whom. This provided me with data which convinced me that patient-held records have an important part to play in cancer care. Most health professionals involved were positive, apart from some reservations about it being yet more time-consuming paperwork. There was some criticism of the records being too complicated, which resulted in a simpler version being designed. Because they were not in common use, or standard in interactions between patients and staff throughout the health district, patients had to be fairly assertive when presenting the records to doctors, nurses and others to fill in. Their requests met with few refusals, if occasional surprise, and most records were filled in with brief details of relevant health interventions.

On a critical note, sometimes the entries were illegible and written in medical jargon instead of patient-friendly language. Some more specific comments which came out of the evaluation were that the records could be particularly useful for out-of-hours doctors visiting patients whose illness and circumstances they do not know. The list of current medication was also felt to be an advantage when patients were seen away from computerised records, as long as they were kept up to date by any doctor who changed any of the drugs.

One of the limitations of the evaluation was that I was only able to contact people whom I knew had used the PHR, which assumes that they were in favour of its use. This could mean that there were people who refused to write in them because of some doubts, uncertainty, antagonism or for other reasons. This has been borne out by patients who in the main comment that they are a good idea, welcomed by most but occasionally meeting resistance. One consultant was reported to have refused, saying she was far too busy to write in the record, which made the patient feel he was being a nuisance. 
Another major failing which happened on more then one occasion was that when a patient was admitted to hospital, the record was placed on a bedside locker until the patient was ready to be discharged when it disappeared, never to be seen again. It would seem that on a busy acute ward, staff feel it is someone else's job to make an entry in the record. This could reflect my inadequate dissemination of information when the records were launched, and not maintaining sufficient contact with wards, where there is a high turnover of staff, in order to keep them informed of any developments.

I felt quite humbled by the way cancer patients have been seen in various health settings, clutching their blue book, and in the way they themselves have painstakingly filled in their own personal comments. Members of one family kept a very detailed record of every aspect of their mother's care in the weeks before she died, which would have put any nursing record to shame. They diligently showed it to every health professional who visited. The wife of another patient who died sent the record back to me with a note asking if I would return it when I had finished with it because it held all the details of the last weeks of her husband's life which she felt she needed, presumably to help her through the grieving process. One personal, probably subjective, observation is that men seem to use the PHR more than women. I can give no explanation for this except the standard theories that this is because men may be more assertive and less intimidated than women or that as women are generally better verbal communicators than men, especially in emotional issues, they do not have the same need to have things in print.

\section{PROBLEMS WITH IMPLEMENTATION}

After the evaluation results were discussed at a cancer steering group meeting, and some modifications made to the records, a decision was made to introduce patient-held records throughout the health authority. This seemed fairly simple at the time, no more than extending the practice to every other GP practice in the Lancaster Priority Services Trust. It involved an attempt to see every GP, district nurse and other members of primary health care teams as well as communicating with hospital staff and all personnel involved with cancer patients: a lot of people, but not an impossible task.

Around that time, the three trusts - Lancaster, South Cumbria and Barrow - merged, which made the implementation of patient-held records a significantly bigger task. At that time too, following the publication of an article describing the Lancaster initiative in the International Journal of Palliative Nursing ${ }^{(5)}$, along with the recommendations of the Calman Report, many other oncology and palliative care units started taking an interest in PHRs. The Marie Curie Centre in Newcastle held a one-day conference on the very subject, highlighting the advantages and disadvantages and describing their attempts to introduce them to three types of patient, ie those with lung, breast and colorectal cancers. Professor Ilora Finlay spoke critically at this conference, stating that, in her experience, a cheap notebook from the newsagent would be just as useful for those patients who wanted everything noted down. The main theme of the day, however, was much more positive.

The decision to give cancer patients their own records was made. Barrow and South Cumbria had no person nominated to help to launch the initiative in their areas. It was assumed that it would just happen. More meetings with various interested parties followed and the practice is now in place. The records are distributed by Macmillan nurses when they first meet a patient with cancer. In the Lancaster health district there has been a limited response and they are often met with apathy. Patients complain that nobody asks for the blue book so they do not feel encouraged to present them.

Last year the accreditation of palliative care services was started as a result of the recommendation of the Calman Report in an attempt to standardise and improve palliative care throughout the country. Morecambe Bay Palliative Care Services was one of the pilots for the accreditation process and one of the recommendations was for the continued growth of the use of PHRs. Palliative care units in other parts of the country will also have been encouraged to introduce them as a result of their own accreditation procedures. As a result, I have been approached by several other areas in the country to speak as an 'expert' on the use of PHRs in cancer care. This seems rather ironic, as since the supposed universal introduction of PHRs throughout Morecambe Bay, the initiative has fallen rather flat. This is due partly to intertia and the poor participation of many health professionals, but is mainly because not enough time, personnel or resources have been available to make it a success. A strategy is required to motivate all those who need to be involved, to inform and educate them and keep the momentum going, repeatedly updating knowledge and advertising the PHRs so that they become a routine part of patient care. When the idea was first broached, it was not anticipated that it would need such a big commitment of time and personnel in order to succeed. In Preston, where I have been asked to advise on their introduction of PHRs, a huge committee whose members include oncology unit staff, community doctors and nurses, pharmacists, managers and many others has been meeting for months to ensure that their introduction goes smoothly and any problems are foreseen and dealt with. I have been minimally involved with their planning, mainly advising them how not to do it.

\section{HOW TO USE THE PATIENT-HELD RECORD}

Although there have been problems with implementation, they are not insurmountable and I would like to take this opportunity to urge anyone who is involved in the health care of cancer patients to record any intervention in the blue book. A simple entry, legible and in plain English, is all that is needed. Computerised data is available in most surgeries these days so laboratory and radiology results are usually accessible; there is, therefore, no need to record such details. Certain important results (for example, a sudden rise in PSA or lytic lesion found on bone scan) should be entered in the PHR, after being given to patients.

One example of good use of the PHR occurred when a 40-year-old woman with ovarian cancer wrote a list of questions regarding her anticipated treatment. These questions were answered in the PHR by the oncologist for her to assimilate at her own pace. Her GP, who saw her after her oncology appointment, learned what treatment she would be getting and what side effects she might expect.

An example of a poor entry was a barely decipherable scrawl from someone, presumably a doctor though there was no name or signature attached, which told of an increase in the dose of steroids. This was not useful information for the patient, not was the medication list altered to inform subsequent users. Whilst it is appreciated that writing records 
is a vital, if onerous, part of our work and that an extra recording can make a big difference to an already busy day, I feel that it is a step forward for patients to hold their own details of care. Traditional medical and nursing case notes serve the needs of healthcare personnel only. It would be a valuable culture change to include the patient.

\section{CONCLUSION}

To summarise, PHRs are a good idea if used properly. A further, more comprehensive, evaluation is planned which should inform us further of flaws in the current system and ways in which they can be overcome. Patients generally greet the introduction of the record with enthusiasm and comment on how useful the records will be. This enthusiasm wanes as they are met with indifference or impatience.

As patients have a legal right to examine their own medical notes, and as access to these traditional notes can become very difficult when patients are seen in more than one setting, it makes sense to have the notes where the patient is. Total commitment is needed from patients and healthcare staff before we can hope for real success. Computerised medical information accessible to health professionals should improve information exchange at one level but this does not involve patients or enhance their part in making decisions about their care.

No-one claims that PHRs will dispel communication problems overnight, but it is hoped that they will go some way to improve the exchange of information, create better understanding of each other's roles among participating healthcare staff, and minimise ambiguity, the duplication of tasks and misunderstandings. Patients should have a greater sense of involvement and ownership of their illness. PHRs should facilitate more honest and open exchanges and encourage partnership in care rather than medicalisation of a condition which already leaves patients feeling that they have lost control and autonomy.

\section{REFERENCES}

1 Calman K, Hine D A policy framework for commissioning cancer services London: Department of Health 1995

2 UKCC Standards for records and record keeping United Kingdom Central Council for Nursing, Midwifery and Health Visiting 1993

3 Buckman R What you really need to know about cancer London: Macmillan 1995

4 Ingham JM, Coyle N Teamwork in end of life care: a nurse/physician perspective on introducing physicians to palliative care concepts In Clark D, Hockley J, Ahmedzai S (eds) New themes in palliative care Buckingham: Open University Press 1997 pp255-274

5 McCann C Communication in cancer care; introducing patient-held records Int J Palliative Nursing 1998;4(5):222-229 